\title{
SPAG6 silencing induces autophagic cell death in SKM-1 cells via the AMPK/mTOR/ULK1 signaling pathway
}

\author{
MENG ZHANG ${ }^{1,2}$, JIE LUO ${ }^{1,2}$, XIAOHUA LUO ${ }^{1}$ and LIN LIU ${ }^{1}$ \\ ${ }^{1}$ Department of Hematology; ${ }^{2}$ Laboratory Research Center, \\ The First Affiliated Hospital of Chongqing Medical University, Yuzhong, Chongqing 400016, P.R. China
}

Received September 29, 2019; Accepted March 6, 2020

DOI: $10.3892 / \mathrm{ol} .2020 .11607$

\begin{abstract}
As a member of the cancer-testis antigen family, sperm-associated antigen 6 (SPAG6) has been reported to be associated with the pathogenesis of myelodysplastic syndromes (MDS). Previous studies have demonstrated that SPAG6 is upregulated in bone marrow from patients with MDS and MDS-transformed acute myeloid leukemia and that knockdown of SPAG6 expression levels suppressed proliferation and promote apoptosis and differentiation in SKM-1 cells. However, the association between SPAG6 and autophagy in SKM-1 cells remains unclear. Hence, the aim of the present study was to investigate this association and its underlying mechanism. The present study used a short hairpin RNA (shRNA) lentivirus to silence SPAG6 expression levels in SKM-1 cells and demonstrated that SPAG6 knockdown increased autophagy and apoptosis. Furthermore, pharmacologically inhibiting autophagy with chloroquine and 3-methyladenine decreased SPAG6 knockdown-mediated apoptosis, indicating that SPAG6 knockdown-mediated autophagy promoted apoptosis in SKM-1 cells. Additionally, compared with the expression levels in negative control-shRNA lentivirus-transfected SKM-1 cells, the protein expression levels of phosphorylated AMP-activated protein kinase (p-AMPK) and phosphorylated unc-51-like autophagy activating kinase 1 (p-ULK1) were upregulated, while phosphorylated mammalian target of rapamycin (p-mTOR) protein expression was downregulated in SPAG6-shRNA lentivirus-transfected cells. Moreover, inhibiting AMPK expression levels with Compound $\mathrm{C}$, a specific inhibitor of AMPK, attenuated SPAG6 knockdown-induced autophagy and apoptosis, suggesting that AMPK-mediated autophagy enhanced the pro-apoptotic effect of SPAG6 knockdown in
\end{abstract}

Correspondence to: Professor Lin Liu, Department of Hematology, The First Affiliated Hospital of Chongqing Medical University, 1 Youyi Road, Yuzhong, Chongqing 400016, P.R. China

E-mail: liul7776@aliyun.com

Key words: sperm-associated antigen 6, myelodysplastic syndromes, autophagy, apoptosis, AMP-activated protein kinase/mTOR/unc-51-like autophagy activating kinase 1 pathway
SKM-1 cells. Taken together, the results of the present study demonstrated that SPAG6 silencing triggered autophagy via regulation of the AMPK/mTOR/ULK1 signaling pathway, which further contributed to the apoptosis of SKM-1 cells induced by SPAG6 knockdown. Thus, the current results indicate that SPAG6 may be a potential therapeutic target against MDS, and that autophagy may represent a potential mechanism for the treatment of MDS.

\section{Introduction}

Myelodysplastic syndromes (MDS) are defined as clonal hematopoietic stem cell (HSC) malignancies with characteristic manifestation of peripheral blood cytopenias due to ineffective hematopoiesis and dysplasia of the bone marrow (BM). MDS has a high risk of conversion to acute myeloid leukemia (AML) (1). Treatments for patients with MDS include hematopoietic therapy, differentiation therapy, demethylation therapy and allogeneic BM HSC transplantation (allo-HSCT) (2). However, the efficacy of these treatments is unsatisfactory. Data from the Center for International Blood and Marrow Transplant Research (CIBMTR) indicated transplant-related mortality (TRM) and relapse rates of 30 and $30 \%$, respectively, following allogeneic HCT from human leukocyte antigen (HLA)-matched related donors; the 3-year probability of survival is $40 \%$ (3). Upon progression to AML, patients exhibit a poor response to standard therapy and a high likelihood of mortality. Therefore, it is urgently necessary to identify effective therapeutic targets to prevent MDS progression.

Sperm-associated antigen 6 (SPAG6), a member of the cancer-testis antigen family, was first discovered in human testis tissue, where its functions include regulating sperm flagella motility and germ cell maturation (4). However, increasing evidence has demonstrated that SPAG6 may influence the progression of certain malignant diseases, including gastric, breast and lung cancer (5). Furthermore, a number of reports have demonstrated that, compared with normal controls, SPAG6 is upregulated in BM from patients with MDS, MDS-AML or de novo AML, and that high expression levels of SPAG6 are associated with poor survival $(6,7)$. SKM-1 is an MDS-AML cell line that was established from the peripheral blood of a male patient with MDS transformation to AML. As there are currently no MDS cell lines, SKM-1 cells are considered to be the optimal model for investigating the 
pathogenesis of MDS (8). Previous studies have demonstrated that lentivirus-mediated SPAG6 knockdown suppresses proliferation and promotes apoptosis and differentiation in SKM-1 cells, confirming that SPAG6 influences the progression of MDS (7,9-11). However, the effects of SPAG6 on autophagy and its underlying mechanisms have not yet been identified.

Autophagy is a physiological process in which unnecessary intracellular materials are degraded by the lysosome for recycling. Therefore, it is essential for the maintenance of cell development, metabolism and cellular homeostasis. However, previous evidence has demonstrated that autophagy exerts both pro-survival or pro-apoptotic functions in disease, depending on the tumor condition, disease progression and cellular microenvironment (12). There is growing evidence that autophagy serves a critical role in the pathogenesis of MDS and AML; autophagy regulates the differentiation and development of HSCs by removing damaged mitochondria and reactive oxygen species (ROS) $(13,14)$. Further studies have indicated that a lack of autophagy in HSCs may facilitate the progression of MDS and AML $(15,16)$. In addition, a recent study demonstrated that, compared with normal controls, autophagy was decreased, and damaged mitochondria and ROS levels were increased in patients with MDS and MDS-AML (17), suggesting that autophagy functions as a tumor suppressor in the progression of MDS. However, the possible cause of decreased autophagy in MDS remains unclear. Thus, studies are required to investigate the mechanisms underlying decreased autophagy in MDS to provide guidance for the prevention and treatment of MDS.

AMP-activated protein kinase (AMPK) is a key factor in regulating cellular energy and metabolism, and serves an essential role in the pro-differentiation and anti-leukemic properties of myeloid malignancies, via regulation of multiple downstream signaling molecules to induce autophagy (18). For example, it directly or indirectly inhibits cancer-promoting mTOR complex 1 (mTORC1) signaling and activates pro-apoptotic p53 $(19,20)$. However, previous studies have demonstrated that the expression levels of autophagy, differentiation and AMPK mRNA were significantly decreased in cells collected from patients with high-risk MDS compared with patients with low-risk MDS or healthy controls $(7,17,18)$. A previous study demonstrated that SPAG6 silencing increases the differentiation of SKM-1 cells (7), which suggests that SPAG6 silencing may regulate differentiation and autophagy in SKM-1 cells via activation of the AMPK signaling pathway. To the best of our knowledge, however, the potential molecular mechanism has not yet been clarified,

The present study demonstrated that SPAG6 knockdown may trigger autophagy and apoptosis in SKM-1 cells and that autophagy inhibitors attenuated SPAG6 knockdown-induced cell apoptosis. Furthermore, the present study investigated the underlying mechanism of SPAG6 knockdown-induced autophagy, which may be mediated by regulation of the AMPK/mTOR/ULK1 signaling pathway.

\section{Materials and methods}

Cell culture and infection. SKM-1, the human MDS-AML cell line, was provided by Professor Jianfeng Zhou of Tongji Medical College of Huazhong University of Science and
Technology (Wuhan, China) -and cultured in RPMI-1640 medium (Corning Inc.) containing 10\% fetal bovine serum (Capricorn Scientific GmbH) without antibiotics. Cells were grown in complete medium with 3-methyladenine (3-MA; $5 \mathrm{mM} / \mathrm{l}$ ), chloroquine (CQ; $10 \mu \mathrm{M} / \mathrm{l})$ or the AMPK inhibitor Compound C (5 $\mu \mathrm{M} / \mathrm{l})$, purchased from Selleck Chemicals and all cells were incubated at $37^{\circ} \mathrm{C}$ with $5 \% \mathrm{CO}_{2}$. Lentiviral short hairpin RNAs (shRNAs) were designed and synthesized by Shanghai GeneChem Co.,Ltd.A total of three different shRNAs for SPAG6 were designed (sequences listed in Table SI) and the shRNA used as the negative control (NC) was 5'-TTCTCC GAACGTGTCACGT-3'. SKM-1 cells at the logarithmic stage were seeded in 24 -well plates $\left(5 \times 10^{4}\right.$ cells/well) before being transfected with SPAG6-shRNA lentivirus or NC-shRNA lentivirus at a multiplicity of infection of 80 in the presence of $5 \mu \mathrm{g} / \mathrm{ml}$ polybrene, an enhancing reagent (Invitrogen; Thermo Fisher Scientific, Inc.). Another $500 \mu 1$ fresh complete RPMI-1640 medium supplemented with 10\% FBS, without antibiotics was added to cells in each group $20 \mathrm{~h}$ later, and the cells were then resuspended in fresh complete medium after an additional $24 \mathrm{~h}$. Three days later, fresh complete medium with puromycin at a concentration of $0.5 \mathrm{mg} / \mathrm{ml}$ was used to culture the transfected cells.

Reverse transcription-quantitative polymerase chain reaction $(R T-q P C R)$. Total RNA was extracted separately from cells treated with NC-shRNA or SPAG6-shRNA using TRIzol reagent (Takara Biotechnology Co., Ltd.); then, the RNA samples were reverse transcribed into cDNA using a PrimeScript RT reagent kit (Takara Biotechnology Co., Ltd.), according to the manufacturer's protocols. qPCR was subsequently performed using a CFX-Connect Real-Time PCR system (Bio-Rad Laboratories, Inc.) and TB Green Master Mix (Takara Biotechnology Co., Ltd.) for a total of 45 cycles, following standard assay procedures. The thermocycling parameters used were $95^{\circ} \mathrm{C}$ for $30 \mathrm{sec}$, followed by 45 cycles at $95^{\circ} \mathrm{C}$ for $5 \mathrm{sec}$ and $62^{\circ} \mathrm{C}$ for $30 \mathrm{sec}$. The mRNA expression levels of SPAG6 and autophagy-associated genes were calculated using the $2^{-\Delta \Delta C q}$ method (21) with GAPDH as an internal control. The primer sequences are listed in Table I.

Western blot analysis and antibodies. Cells from each group were collected and washed with PBS three times. Then, all cells were lysed in RIPA lysis buffer (Beyotime Institute of Biotechnology) containing PMSF and phosphatase inhibitor (Boster Biological Technology). A bicinchoninic acid kit (Beyotime Institute of Biotechnology) was used to measure protein concentrations, according to the manufacturer's protocol. Total cellular proteins $(40 \mu \mathrm{g})$ in each lane were separated via SDS-PAGE on 10 or $12 \%$ gels and transferred onto PVDF membranes (Merck KGaA) (12\% gel was used for LC3; 10\% gel was used for SPAG6, P62, Beclin1, ATG5, ATG7, cleaved caspase 3, Bcl-2, Bax, p-AMPK, AMPK, p-mTOR, mTOR, p-ULK1, ULK1 and GAPDH). Then, the membranes were blocked with 5\% skimmed milk at room temperature for $2 \mathrm{~h}$ before incubation with the corresponding primary antibodies overnight at $4^{\circ} \mathrm{C}$. The next day, the membranes were incubated with goat anti-rabbit secondary antibody (cat. no. A0216; 1:3,000; Beyotime Institute of Biotechnology) for $1 \mathrm{~h}$ at room temperature. Finally, the bands 
Table I. Primer sequences used for RT-qPCR analysis

\begin{tabular}{lll}
\hline Gene & \multicolumn{1}{c}{ Forward $\left(5^{\prime}-3^{\prime}\right)$} & \multicolumn{1}{c}{ Reverse $\left(5^{\prime}-3^{\prime}\right)$} \\
\hline GAPDH & CTTTGGTATCGTGGAAGGACTC & GTAGAGGCAGGGATGATGTTCT \\
SPAG6 & AGTGCGACATTCTTCCACAGCTTG & GCGTATCCAGTGCTCCACAATCG \\
LC3 & CCGACTTATTCGAGAGCAGCATCC & GTCCGTTCACCAACAGGAAGAAGG \\
Beclin1 & ATCTAAGGAGCTGCCGTTATAC & CTCCTCAGAGTTAAACTGGGTT \\
ATG5 & GATGGGATTGCAAAATGACAGA & GAAAGGTCTTTCAGTCGTTGTC \\
ATG7 & TGTATAACACCAACACACTCGA & GGCAGGATAGCAAAACCAATAG
\end{tabular}

were visualized using an enhanced chemiluminescence kit (Advansta, Inc.), and the relative protein expression levels were analyzed using Fusion FX Spectra software (version 7; Vilber Lourmat). The primary antibodies were as follows: SPAG6 (cat. no. HPA038440; 1:1,000; Sigma-Aldrich; Merck KGaA); GAPDH (cat. no. AG019, 1:1,500 Beyotime Institute of Biotechnology); Beclin1, P62, cleaved caspase-3, Bcl-2, Bax, mTOR and phosphorylated (p-)mTOR (cat. nos. ab210498, ab109012, ab2302, ab32124, ab32503, ab32028 and ab109268, respectively; all 1:1,000; all Abcam); LC3, ATG5, ATG7, AMPK, p-AMPK, ULK1 and p-ULK1 (cat. nos. 12741, 12994, 8558, 2532, 2535, 8054 and 5869, respectively; all 1:1,000; all Cell Signaling Technology, Inc.).

Transmission electron microscopy. Cells treated with NC-shRNA or SPAG6-shRNA alone, as well as cells co-treated with SPAG6-shRNA and 3-MA or CQ were collected, centrifuged at $350 \mathrm{x}$ g for $5 \mathrm{~min}$ at room temperature and subsequently fixed with $2.5 \%$ glutaraldehyde and $1 \%$ osmic acid for $2 \mathrm{~h}$ at $4^{\circ} \mathrm{C}$. Next, the prepared cells were washed twice with ddH2O and embedded following dehydration with acetone. Then, the samples were solidified and cut into $1-\mu \mathrm{m}$ sections, which were double-stained with uranium acetate for $10 \mathrm{~min}$ and lead citrate for $30 \mathrm{~min}$, at $35^{\circ} \mathrm{C}$. Sections were observed under a Hitachi-7500 transmission electron microscope (Hitachi, Ltd.; magnification, x20,000).

Flow cytometry. Cells treated with NC-shRNA or SPAG6-shRNA alone and cells co-treated with SPAG6-shRNA and 3-MA or CQ, as well as cells co-treated with Compound C and NC-shRNA or SPAG6-shRNA were collected and washed with PBS twice before being resuspended in $500 \mu 1 \mathrm{PBS}$. Then, the cells were double-stained with annexin V APC and DAPI (BD Pharmingen; BD Biosciences) and incubated in the dark at room temperature for $10 \mathrm{~min}$. The fluorescence intensities of the cells were examined immediately by flow cytometry (CytoFLEX, Beckman Coulter, Inc.).

Statistical analysis. SPSS (version 24.0; IBM Corp.) and GraphPad Prism software (version 8.0; GraphPad Software, Inc.) were used to analyze all data. Data are presented as the mean \pm standard deviation. An unpaired Student's t-test was used for two-group analysis and a One-way analysis of variance was used for comparisons among multiple groups, followed by the Newman-Keuls post hoc test. All experiments were performed in triplicate. $\mathrm{P}<0.05$ was considered to indicate a statistically significant difference.

\section{Results}

SPAG6 silencing induces autophagy in SKM-1 cells. To the best of our knowledge, there is no previous evidence concerning the association between SPAG6 and autophagy in MDS. It is well known that the expression of autophagy associated genes (ATG) increases while the expression of autophagy receptors decreases when autophagy is activated (22). LC3 is a homologue of ATG8, including two forms of LC3-I and LC3-II, the latter being a marker of autophagy; Beclin1 is a homologue of ATG6; these, as well as ATG5 and ATG7 are autophagy associated genes (22). P62 acts as an autophagy receptor, fusing autophagosomes (including cargo) with lysosomes, and then P62 and cargo are degraded by autophagolysosomes, resulting in a decrease in P62 expression levels (22). Therefore, in order to investigate whether SPAG6 is associated with autophagy in SKM-1 cells, cells were transfected with shRNAs. The optimal shRNA for SPAG6 was screened via RT-qPCR and western blot analysis, and was identified as SPAG6-shRNA3 (Fig. S1). Following the suppression of the expression levels of SPAG6, the expression levels of autophagy-related genes were analyzed: Compared with its expression levels in SKM-1 cells transfected with NC-shRNA lentivirus, SPAG6 mRNA and protein expression levels in cells transfected with SPAG6-shRNA lentivirus were significantly decreased. Moreover, there was an increase in mRNA and protein expression levels of LC3-II, Beclin1, ATG5 and ATG7, and a decrease in p62 (Fig. 1A and B). Transmission electron microscopy is considered to be the gold standard for observing autophagosome formation. In the present study, a significant increase in autophagosome formation was detected in the SPAG6 knockdown group (Fig. 1C and D). Taken together, the results of the present study indicate that SPAG6 knockdown may induce autophagy in SKM-1 cells.

SPAG6 knockdown triggers apoptosis in SKM-1 cells. The present study used western blot analysis and flow cytometry to detect the apoptosis rate in each group. Compared with the NC-shRNA group, Bax and cleaved caspase-3 protein levels were significantly increased in the SPAG6-shRNA group, whereas Bcl-2 protein levels were decreased (Fig. 2A). These data indicated that SPAG6 knockdown significantly induced cell apoptosis. This was confirmed using flow cytometry (Fig. 2B and C). Overall, these results indicated that SPAG6 silencing may induce apoptosis in SKM-1 cells, which is consistent with previous evidence (9-11). 

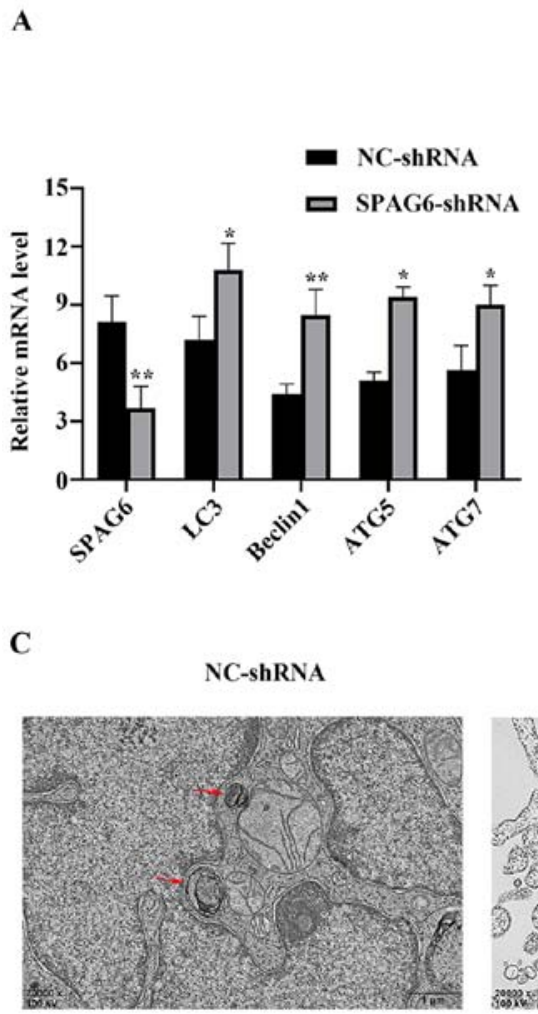

B
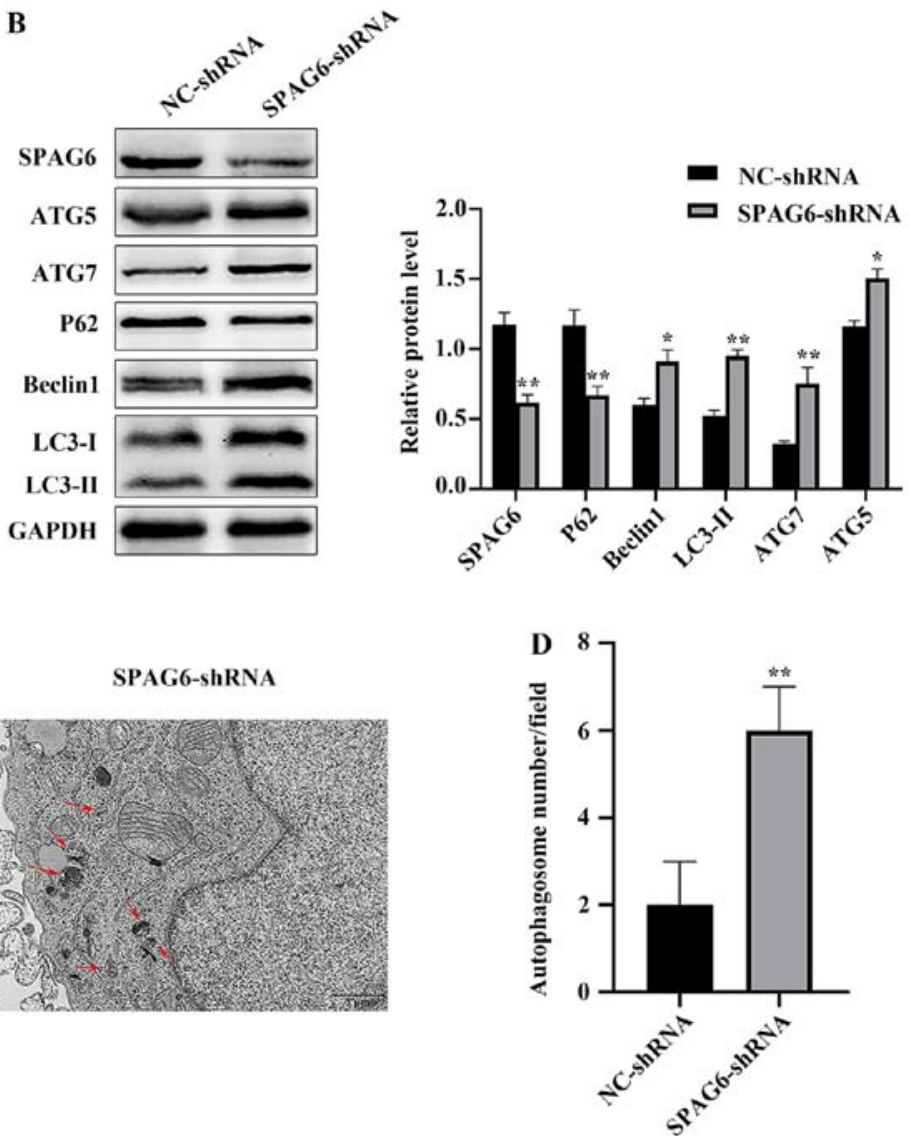

Figure 1. SPAG6 silencing induces autophagy in SKM-1 cells. (A) Relative mRNA expression levels of SPAG6, LC3, Beclin1, ATG5 and ATG7 in each group were detected using RT-qPCR. (B) Relative protein expression levels of SPAG6, LC3, Beclin1, p62, ATG5 and ATG7 in each group were detected using western blot analysis. GAPDH was used as a loading control. (C) Transmission electron microscopy revealed the intracellular ultrastructures in each group (magnification, x20,000). The images display a representative experiment from three independent experiments. Red arrows indicate autophagic vacuoles. Scale bar, $1 \mu \mathrm{m}$. (D) Number of autophagosomes per field was quantified. Data are presented as the mean \pm standard deviation of three independent experiments. ${ }^{*} \mathrm{P}<0.05 ;{ }^{* *} \mathrm{P}<0.01$ vs. NC-shRNA. NC, negative control; shRNA, short hairpin RNA; SPAG6, sperm-associated antigen 6; LC3, microtubule-associated protein 1 light chain 3; Beclin1, autophagy-associated protein 6; p62, sequestosome 1; ATG5, autophagy-related protein 5; ATG7, autophagy-related protein 7.

Inhibition of autophagy attenuates SPAG6 knockdowninduced apoptotic cell death in SKM-1 cells. Autophagy activation has previously been reported to induce apoptosis in certain types of human cancer $(23,24)$. Thus, the present study used the autophagy inhibitors 3-MA and CQ to block autophagy in order to investigate the effect of autophagy on apoptosis induced by SPAG6 silencing. It has previously been reported that 3-MA and CQ inhibit autophagy at different stages: 3-MA inhibits autophagy at an early stage, resulting in decreased LC3-II and Beclin1 expression levels, and increased P62 expression levels (25). However, CQ suppresses autophagy by inhibiting the degradation of autophagy lysosomes, which results in additional LC3-II and Beclin1 accumulation and decreased p62 degradation (26). The present study demonstrated that, compared with the expression levels in cells transfected with SPAG6-shRNA alone, LC3-II and Beclin1 protein expression levels were significantly decreased in SKM-1 cells cotreated with SPAG6-shRNA/3-MA, while P62 protein expression levels were increased; these changes were accompanied by decreased cleaved caspase- 3 and Bax protein expression levels and increased $\mathrm{Bcl}-2$ protein expression levels (Fig. 3A and D). Furthermore, SKM-1 cells treated with SPAG6-shRNA/CQ yielded the same results, except increased LC3-II and Beclin1 protein expression levels (Fig. 3A and D).
In addition, the number of autophagosomes was notably decreased in SPAG6-shRNA/3-MA-cotreated SKM-1 cells but was increased in SKM-1 cells treated with SPAG6-shRNA/CQ compared with cells transfected with SPAG6-shRNA alone (Fig. 3B and C). Furthermore, the present study used flow cytometry to analyze the effects of 3-MA and CQ on apoptosis. Levels of annexin V APC/DAPI positive cells were decreased in cells co-treated with SPAG6-shRNA and 3-MA or CQ compared with cells treated with SPAG6-shRNA alone (Fig. 3E and F). Collectively, the current results indicate that inhibiting autophagy with 3-MA and CQ may attenuate SPAG6 knockdown-mediated apoptosis in SKM-1 cells.

SPAG6 knockdown induces autophagy via the AMPK/mTOR/ULK1 pathway. There is evidence to indicate that autophagy may be induced via the AMPK/mTOR/ULK1 pathway $(27,28)$. In order to investigate whether this pathway participates in SPAG6 knockdown-mediated autophagy in SKM-1 cells, the present study detected AMPK, p-AMPK, ULK1, p-ULK1, mTOR and p-mTOR protein levels using western blotanalysis. As demonstrated in Fig. 4A, although the total AMPK, ULK1 and mTOR protein levels were not altered, p-AMPK and p-ULK1 protein levels were notably increased following SPAG6 knockdown, while the p-mTOR 
A


B
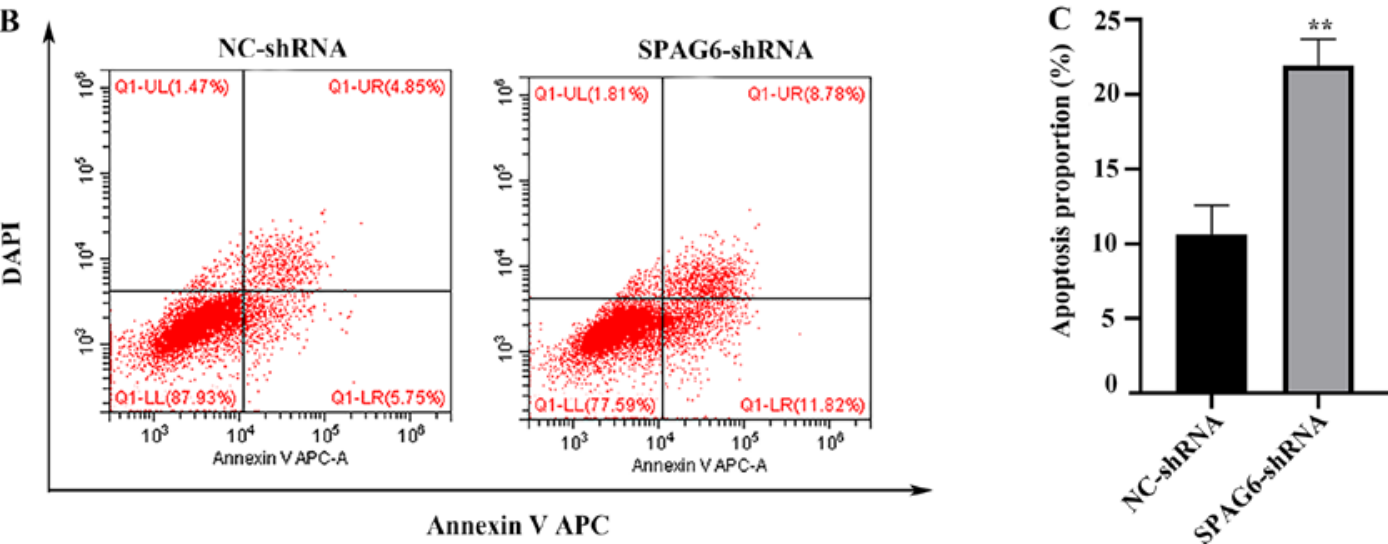

Annexin V APC

Figure 2. SPAG6 knockdown triggers apoptosis in SKM-1 cells. (A) Relative protein expression levels of SPAG6, Bcl-2, Bax and cleaved caspase-3 in each group were detected using western blot analysis. (B) After annexin V APC/DAPI double-staining, the total apoptosis rate of SKM-1 cells treated with NC-shRNA or SPAG6-shRNA was detected by flow cytometry. The images display a representative experiment from three independent experiments. (C) Analysis of the apoptotic rate shown in (B). Data are presented as the mean \pm standard deviation of three independent experiments. ${ }^{* *} \mathrm{P}<0.01 \mathrm{vs}$. NC-shRNA. NC, negative control; shRNA, short hairpin RNA; SPAG6, sperm-associated antigen 6; APC, allophycocyanin; LL, lower left; UL, upper left; LR, lower right; UR, upper right.

protein level was significantly decreased; these changes were accompanied by increases in protein levels of LC3-II, Beclin1, cleaved caspase- 3 and Bax, and decreases in protein levels of P62 and Bcl-2 (Fig. 4A and B). However, compared with the levels in cells transfected with SPAG6-shRNA alone, p-AMPK and $\mathrm{p}-\mathrm{ULK} 1$ protein levels were decreased, whereas the p-mTOR protein level was increased in cells co-treated with SPAG6 and Compound C. Furthermore, the protein level of LC3-II was significantly decreased, indicating that autophagy was suppressed via AMPK inhibition (Fig. 4A and B). Moreover, annexin V APC/DAPI double-staining analysis revealed fewer apoptotic cells among those co-treated with SPAG6-shRNA and Compound C compared with those treated with SPAG6-shRNA alone (Fig. 4C and D). Taken together, the current findings indicate that SPAG6 silencing induced autophagy via the AMPK/mTOR/ULK1 signaling pathway.

\section{Discussion}

A number of studies have demonstrated that chromosomal abnormalities and aberrant gene expression levels serve essential roles in the evolution and progression of MDS $(29,30)$. It has been reported that SPAG6 is upregulated in patients with MDS and AML and that it may regulate apoptosis, proliferation and differentiation in SKM-1 cells (9-11). However, to the best of our knowledge, there are currently no data concerning the role of SPAG6 in autophagy in SKM-1 cells. The present study demonstrated that SPAG6 silencing induced autophagy and apoptosis in SKM-1 cells and that pharmacologically inhibiting autophagy decreased SPAG6 knockdown-induced apoptosis. Furthermore, the present study demonstrated that SPAG6 downregulation mediated p-AMPK and p-ULK1 activation and that blocking the AMPK/mTOR/ULK1 signaling pathway attenuated SPAG6 knockdown-induced autophagy and apoptosis. Hence, the present study indicated that SPAG6 silencing induced autophagy, which further promoted apoptosis in SKM-1 cells, and that the AMPK/mTOR/ULK1 signaling pathway was involved in regulating autophagy.

As a major mechanism of cell component degradation and recycling, autophagy is indispensable in the maintenance of cell homeostasis and metabolism. However, dysfunction of autophagy is involved in a number of pathological conditions that may lead to tumorigenesis. Previous studies have shown that abnormal gene expression levels are important in regulating autophagy in tumor cells $(15,31)$. For example, mice lacking the autophagy-associated gene ATG7 in HSCs develop atypical hyperplasia in the BM, which is similar to MDS and can progress to AML (32). In bladder cancer cells, 




B



SPAG6-ShRNA+3-MA

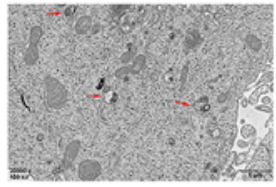

D

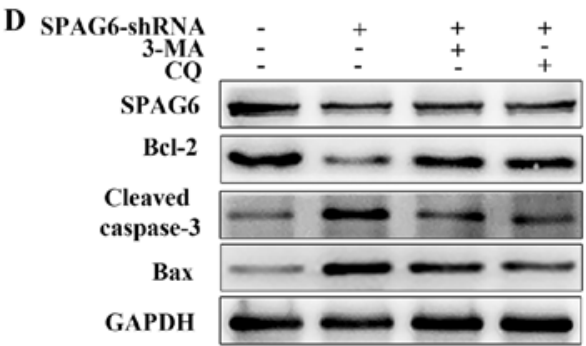

$\mathbf{E}$

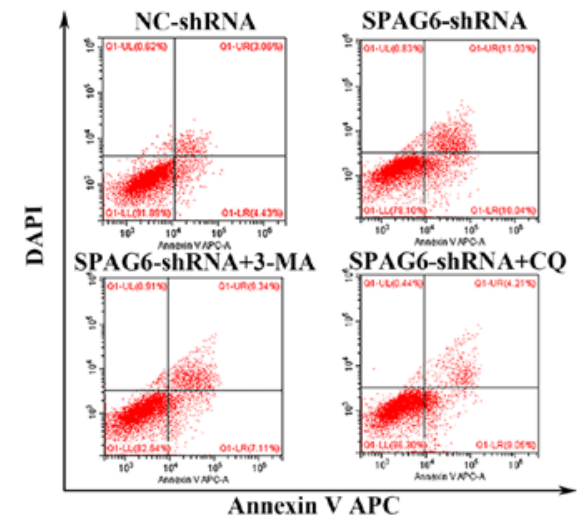

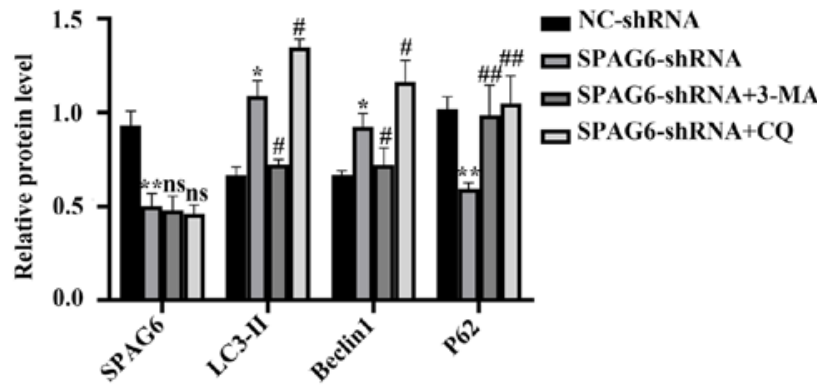

C
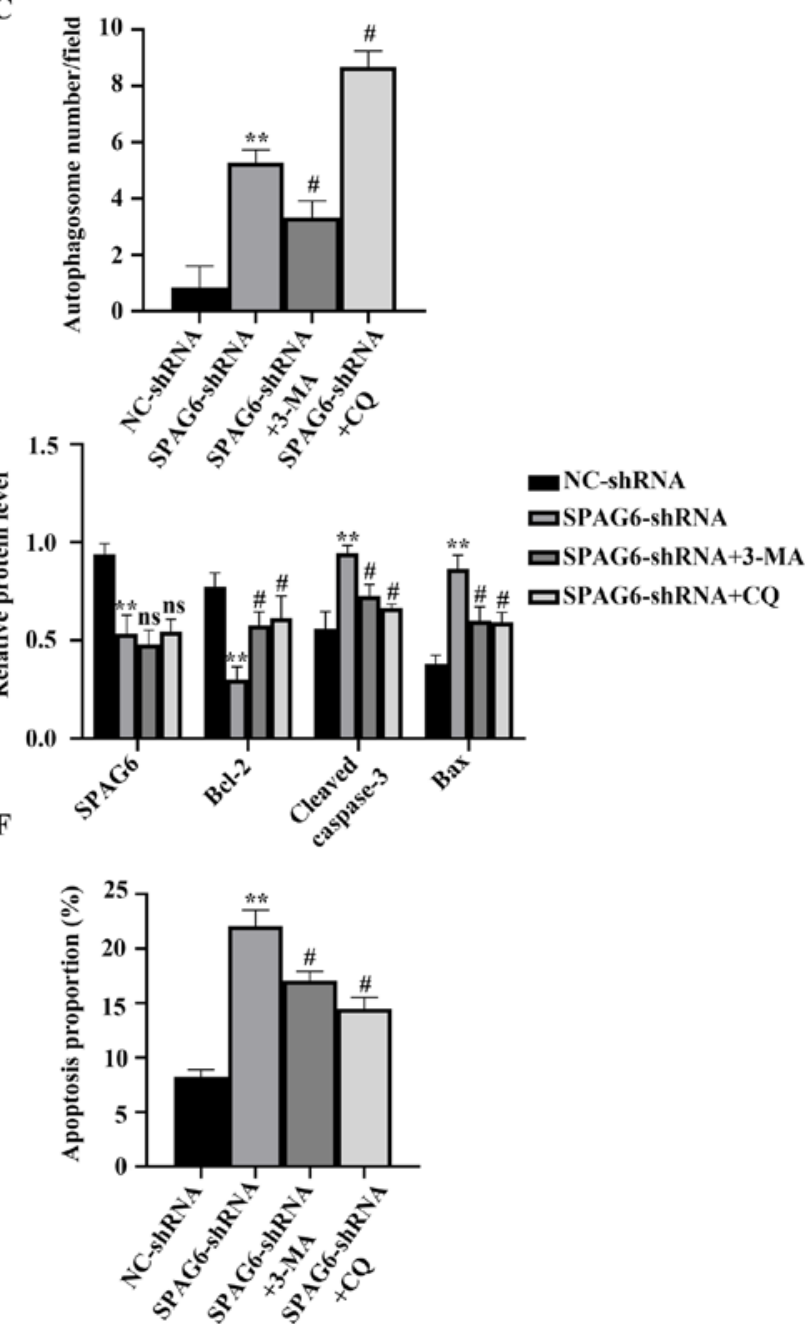

Figure 3. Inhibiting autophagy with autophagy inhibitors attenuates SPAG6 knockdown-induced apoptosis. Cells were treated with SPAG6-shRNA lentivirus or NC-shRNA lentivirus alone, or co-treated with 3-MA or CQ and SPAG6-shRNA. (A) Relative protein expression levels of SPAG6, LC3, Beclin1 and P62 in each group were detected via western blot analysis. (B) Transmission electron microscopy revealed the intracellular ultrastructures of each group (magnification, x20,000). The images display a representative experiment from three independent experiments. Red arrows indicate autophagic vacuoles. Scale bar, $1 \mu \mathrm{m}$. (C) Number of autophagosomes per field was quantified. (D) Relative protein expression levels of SPAG6, Bcl-2, Bax and cleaved caspase-3 in each group were detected using western blot analysis. (E) Following annexin V APC/DAPI double-staining, the total apoptosis rate of SKM-1 cells treated with NC-shRNA or SPAG6-shRNA alone and SKM-1 cells co-treated with SPAG6-shRNA and 3-MA or CQ was detected using flow cytometry. The images display a representative experiment from three independent experiments. (F) Analysis of the apoptotic rate shown in (E) Data are presented as the mean \pm standard deviation of three independent experiments. ${ }^{*} \mathrm{P}<0.05 ;{ }^{* *} \mathrm{P}<0.01$ vs. NC-shRNA, ${ }^{\#} \mathrm{P}<0.05 ;{ }^{\# \#} \mathrm{P}<0.01$ vs. SPAG6-shRNA. NC, negative control; shRNA, short hairpin RNA; SPAG6, sperm-associated antigen 6; LC3, microtubule-associated protein 1 light chain 3; Beclin1, autophagy-associated protein 6; p62, sequestosome 1; CQ, chloroquine; 3-MA, 3-methyladenine; APC, allophycocyanin; LL, lower left; UL, upper left; LR, lower right; UR, upper right; ns, not significant.

G9a inhibition induces autophagy, which further results in autophagic cell death (31). It has been reported that SPAG6, an oncogene that is aberrantly upregulated in patients with MDS and AML, may regulate proliferation, apoptosis and differentiation in SKM-1 cells (7,9-11). The present study used a shRNA lentiviral vector to knockdown SPAG6 expression levels in order to investigate the impact of SPAG6 on autophagy in SKM-1 cells. It was demonstrated that lentivirus-mediated SPAG6 knockdown induced autophagy in SKM-1 cells, as evidenced by an increase in autophagosome formation (detected using transmission electron microscopy) and increases in LC3-II and Beclin1 protein expression levels, 
A
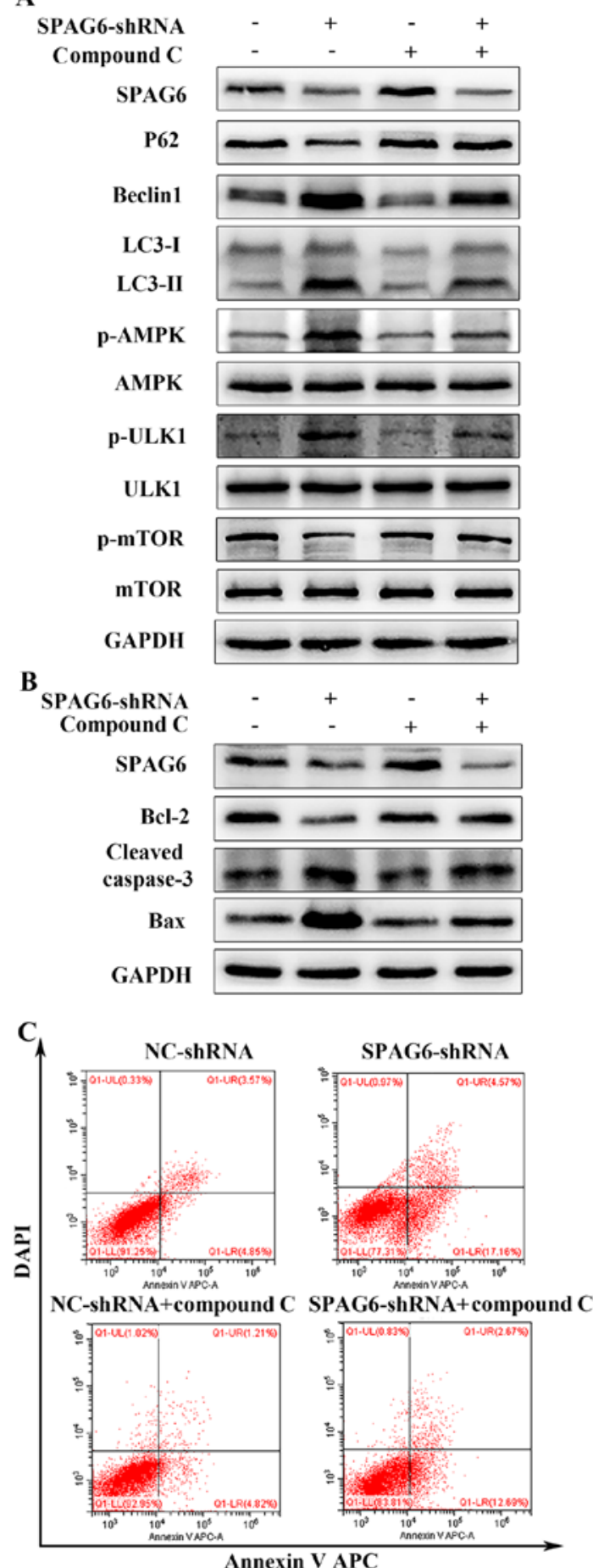

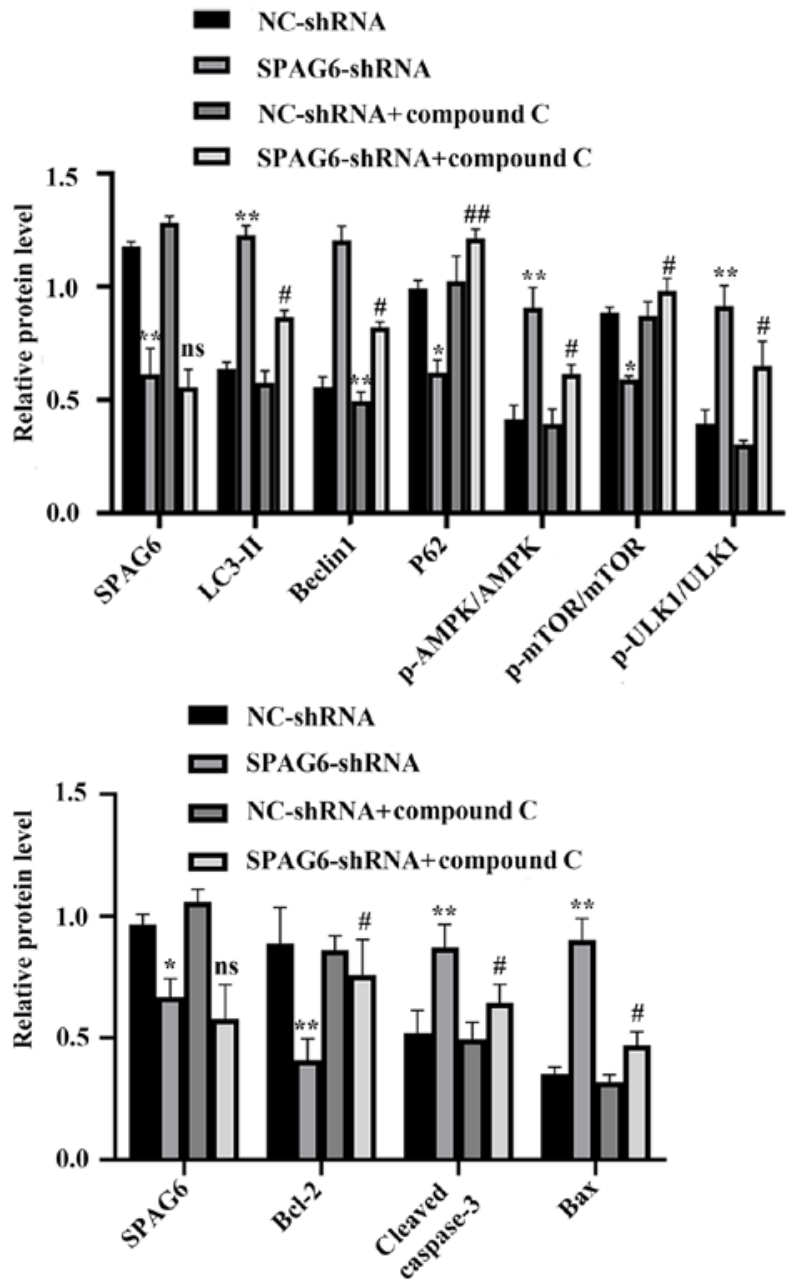

D

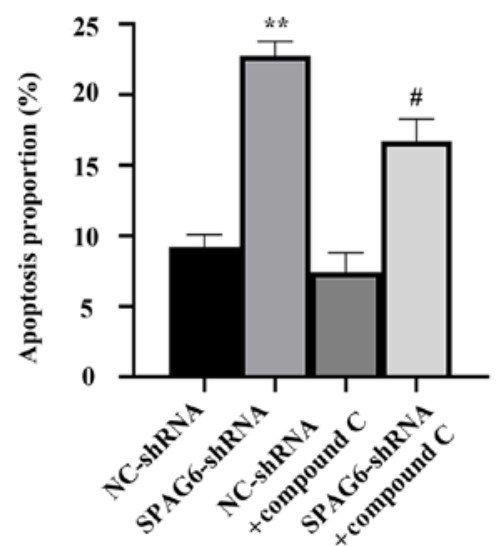

Figure 4. Inhibition of AMPK by Compound C attenuates SPAG6 knockdown-mediated autophagy and apoptosis in SKM-1 cells. Cells were treated with SPAG6-shRNA lentivirus or NC-shRNA lentivirus alone, or co-treated with Compound C and NC-shRNA lentivirus or SPAG6-shRNA lentivirus. (A) Relative protein expression levels of SPAG6, LC3, Beclin1, P62, p-AMPK, p-ULK1 and p-mTOR in each group were detected using western blot analysis. (B) Relative protein expression levels of SPAG6, Bcl-2, Bax and cleaved caspase-3 in each group were detected using western blot analysis. (C) Following annexin $\mathrm{V}$ APC/DAPI double-staining, the total apoptosis rate of SKM-1 cells treated with NC-shRNA or SPAG6-shRNA alone and SKM-1 cells cotreated with Compound C and NC-shRNA or SPAG6-shRNA was detected using flow cytometry. The images display a representative experiment from three independent experiments. (D) Analysis of the apoptotic rate shown in (C). Data are presented as the mean \pm standard deviation of three independent experiments. " $\mathrm{P}<0.05$; ${ }^{* *} \mathrm{P}<0.01$ vs. NC-shRNA, ${ }^{\#} \mathrm{P}<0.05 ;{ }^{\# \#} \mathrm{P}<0.01$ vs. SPAG6-shRNA. NC, negative control; shRNA, short hairpin RNA; SPAG6, sperm-associated antigen 6; LC3, microtubule-associated protein 1 light chain 3; Beclin1, autophagy-associated protein 6; p62, sequestosome 1; p, phosphorylated; AMPK, AMP-activated protein kinase; ULK1, unc-51-like autophagy activating kinase; APC, allophycocyanin; LL, lower left; UL, upper left; LR, lower right; UR, upper right; ns, not significant.

and a decrease in p62 protein expression level (detected using western blot analysis). Previous studies have indicated that SPAG6 regulates differentiation, proliferation, apoptosis and drug sensitivity in neurons and MDS (7,9-11,33,34). To the best of our knowledge, this is the first study to provide evidence for the role of SPAG6 in regulating autophagy in SKM-1 cells. 
The present study investigated the association between SPAG6 and apoptosis in SKM-1 cells, as growing evidence has demonstrated that abnormal proliferation and apoptosis are related to the pathogenesis of MDS. Previous studies have demonstrated that lentiviral transfection-mediated knockdown of SPAG6 inhibits the proliferation of SKM-1 cells, which may be initiated via the AKT/FOXO/p27Kip1 pathway (7,9). In addition, SPAG6 downregulation significantly increases apoptosis in SKM-1 cells, and is accompanied by a significant increase in tumor suppressor genes (P53 and PTEN) (9). Further studies have indicated that SPAG6 knockdown-induced apoptosis in SKM-1 cells may occur via a caspase-independent mechanism, such as PTEN/PI3K/AKT pathway activation or TNF-related apoptosis-inducing ligand pathway induction $(10,11)$. In accordance with these findings, the present study confirmed that lentivirus-mediated SPAG6 knockdown increased apoptosis in SKM-1 cells.

Autophagic cell death and apoptosis are both types of programmed cell death. The association between autophagy and apoptosis is reported to be complicated because molecules that affect apoptosis also play an important role in autophagy activation. For example, downregulating Bcl-2 may induce autophagy by decreasing its binding affinity to the $\mathrm{BH} 3$ domain of Beclin. Subsequently, autophagy activation may increase/decrease apoptosis or sensitivity to chemotherapeutic drugs (35-37). Additionally, autophagy can be both cytotoxic and cytoprotective; previous studies have demonstrated that the autophagy inhibitor 3-MA decreases apoptosis triggered by ATG3 overexpression in SKM-1 cells (36), and in non-small cell lung cancer cells, scutellaria radix-triggered apoptosis is attenuated by autophagy inhibitors (23), which indicates that autophagy has a cytotoxic role in carcinoma treatment. By contrast, certain studies have demonstrated that the autophagy inhibitor CQ enhances apoptosis in SKM-1 cells with long-term exposure to azacytidine (37), and that in ovarian cancer cells, inhibition of autophagy increases paeonol-induced apoptosis (38), which indicates that autophagy may exert a pro-survival role in cancer cells. In order to investigate the association between autophagy and apoptosis in SKM-1 cells, the present study applied the autophagy inhibitors 3-MA and CQ and demonstrated that inhibition of autophagy significantly decreased SPAG6 knockdown-induced apoptosis in SKM-1 cells. This was determined by the decreased number of annexin V APC/DAPI-positive cells and apoptosis-associated protein expression levels, which were detected using flow cytometry and western blot analysis, respectively. Collectively, the results of the present study indicate that SPAG6 knockdown-mediated autophagy functions as a cytotoxic mechanism, which may further induce apoptosis in SKM-1 cells. However, the underlying mechanism of SPAG6 knockdown-mediated autophagy in regulating apoptosis in SKM-1 cells remains unclear. It has been reported that the regulation of apoptosis by autophagy involves numerous mechanisms, such as the Bcl-2 homology-3 domain of Beclin1 and Bcl-2, P62 and PI3K/PTEN/AKT/mTOR $(35,39,40)$. In the present study, SPAG6 knockdown increased Beclin1 expression levels in SKM-1 cells. Thus, it was hypothesized that Beclin1 activation participated in regulating apoptosis via SPAG6 knockdown-mediated autophagy in SKM-1 cells. Further studies are required in order to identify the underlying molecular mechanisms involved in the interaction between autophagy and apoptosis both in vivo and in vitro.

A number of signaling pathways have been reported to regulate autophagy, including the AMPK and the PI3K/AKT/mTOR pathways $(41,42)$. The role of AMPK in regulating differentiation and autophagy in HSCs has been a focus of recent research. Mounting evidence has confirmed that autophagy may be regulated by AMPK, which is activated by the tumor suppressor liver kinase B1 and calcium/calmodulin-dependent protein kinase kinase 2; then, activated AMPK stimulates autophagy by directly phosphorylating and activating ULK1 at Ser555, or by inhibiting mTORC1, which further upregulates p-ULK1 and ATG13 $(27,43)$. The present study demonstrated that, compared with levels in NC-shRNA lentivirus transfected-SKM-1 cells, LC3-II, Beclin1, p-AMPK and p-ULK1 protein levels increased in cells that were transfected with SPAG6-shRNA lentivirus, whereas p62 and p-mTOR protein levels were significantly decreased. Furthermore, inhibition of the AMPK/mTOR/ULK1 signaling pathway using Compound $\mathrm{C}$ significantly decreased autophagy, which further decreased the cytotoxicity mediated by SPAG6 knockdown. Collectively, the results of the present study demonstrated that SPAG6 knockdown induced autophagy via the AMPK/mTOR/ULK1 axis. In addition, these findings are consistent with a recent report demonstrating that iron overload induces autophagy via mitochondrial dysfunction, ROS accumulation and activation of the AMPK signaling pathway in mesenchymal stromal cells from patients with MDS (44), indicating that the AMPK signaling pathway regulates autophagy in MDS cells. It has been reported that the Nod-like receptor protein 3 (NLRP3) inflammasome is activated in patients with MDS and plays a key role in driving the progress of MDS phenotype (45). As a key regulator in NLRP3-dependent inflammation and immunity, AMPK may negatively regulate the activation of the NLRP3 inflammasome, indicating an inverse association between them (46). The present study demonstrated that knockdown of SPAG6 triggered autophagy via the activation of AMPK, which further increased apoptosis in SKM-1 cells. It was hypothesized that SPAG6 mediates AMPK downregulation, which further activates the NLRP3 inflammasome during the progression of MDS. This must be verified by future studies. In addition, a previous study demonstrated that SPAG6 knockdown increased the differentiation level in SKM-1 cells (7). Therefore, further research is required in order to determine whether SPAG6 knockdown-mediated activation of the AMPK signaling pathway participates in this molecular mechanism.

In conclusion, the present study indicated that SPAG6 knockdown induced autophagy via the AMPK/mTOR/ULK1 signaling pathway in SKM-1 cells, and that inhibiting autophagy decreased SPAG6 knockdown-mediated apoptosis. The results of the present study contribute to a better understanding of the mechanisms underlying the role of SPAG6 in the tumor microenvironment and further demonstrate that SPAG6 may be a potential target to attenuate the progression of MDS.

\section{Acknowledgements}

The authors would like to thank Dr Lixue Chen and Dr Xiaojuan Deng (The First Affiliated Hospital of Chongqing 
Medical University, Chongqing, China) for providing technical support.

\section{Funding}

The present study was supported by funds from the National Natural Sciences Foundation of China (grant no. 81570109) and the Natural Science Foundation of Chongqing (grant no. CSTC2013jjB0145).

\section{Availability of data and materials}

The datasets used or analyzed during the current study are available from the corresponding author on reasonable request.

\section{Authors' contributions}

MZ designed and performed the experiments and wrote the manuscript. JL helped to analyze the data. XL contributed to the conception of the study. LL contributed to the study design and provided financial support. All authors read and approved the final version of the manuscript.

\section{Ethics approval and consent to participate}

Not applicable.

\section{Patient consent for publication}

Not applicable.

\section{Competing interests}

The authors declare that they have no competing interests.

\section{References}

1. Corey SJ, Minden MD, Barber DL, Kantarjian H, Wang JC and Schimmer AD: Myelodysplastic syndromes: The complexity of stem-cell diseases. Nat Rev Cancer 7: 118-129, 2007.

2. Greenberg PL, Stone RM, Al-Kali A, Barta SK, Bejar R, Bennett JM, Carraway H, De Castro CM, Deeg HJ, DeZern AE, et al: Myelodysplastic syndromes, Version 2.2017, NCCN clinical practice guidelines in oncology. J Natl Compr Canc Netw 15: 60-87, 2017.

3. Saber W, Cutler CS, Nakamura R, Zhang MJ, Atallah E, Rizzo JD, Maziarz RT, Cortes J, Kalaycio ME and Horowitz MM: Impact of donor source on hematopoietic cell transplantation outcomes for patients with myelodysplastic syndromes (MDS). Blood 122: 1974-1982, 2013.

4. Sapiro R, Kostetskii I, Olds-Clarke P, Gerton GL, Radice GL and Strauss III JF: Male infertility, impaired sperm motility, and hydrocephalus in mice deficient in sperm-associated antigen 6. Mol Cell Biol 22: 6298-6305, 2002.

5. Silina K, Zayakin P, Kalnina Z, Ivanova L, Meistere I, Endzeliņ̌s E, Abols A, Stengrēvics A, Leja M, Ducena K, et al: Sperm-associated antigens as targets for cancer immunotherapy: Expression pattern and humoral immune response in cancer patients. J Immunother 34: 28-44, 2011.

6. Steinbach D, Schramm A, Eggert A, Onda M, Dawczynski K, Rump A, Pastan I, Wittig S, Pfaffendorf N, Voigt A, et al: Identification of a set of seven genes for the monitoring of minimal residual disease in pediatric acute myeloid leukemia. Clin Cancer Res 12: 2434-2441, 2006

7. Jiang M, Chen Y, Deng L, Luo X, Wang L and Liu L: Upregulation of SPAG6 in myelodysplastic syndrome: Knockdown inhibits cell proliferation via AKT/FOXO signaling pathway. DNA Cell Biol 38: 476-484, 2019.
8. Nakagawa T, Matozaki S, Murayama T, Nishimura R, Tsutsumi M, Kawaguchi R, Yokoyama Y, Hikiji K, Isobe T and Chihara K: Establishment of a leukaemic cell line from a patient with acquisition of chromosomal abnormalities during disease progression in myelodysplastic syndrome. Br J Haematol 85: 469-476, 1993.

9. Yang B, Wang L, Luo X, Chen L, Yang Z and Liu L: SPAG6 silencing inhibits the growth of the malignant myeloid cell lines SKM-1 and K562 via activating p53 and caspase activation-dependent apoptosis. Int J Oncol 46: 649-656, 2015.

10. Li X, Yang B, Wang L, Chen L, Luo X and Liu L: SPAG6 regulates cell apoptosis through the TRAIL signal pathway in myelodysplastic syndromes. Oncol Rep 37: 2839-2846, 2017.

11. Yin J, Li X, Zhang Z, Luo X, Wang L and Liu L: SPAG6 silencing induces apoptosis in the myelodysplastic syndrome cell line SKM-1 via the PTEN/PI3K/AKT signaling pathway in vitro and in vivo. Int J Oncol 53: 297-306, 2018.

12. White E and DiPaola RS: The double-edged sword of autophagy modulation in cancer. Clin Cancer Res 15: 5308-5316, 2009.

13. Mortensen M, Ferguson DJ, Edelmann M, Kessler B, Morten KJ, Komatsu $\mathrm{M}$ and Simon AK: Loss of autophagy in erythroid cells leads to defective removal of mitochondria and severe anemia in vivo. Proc Natl Acad Sci USA 107: 832-837, 2010.

14. Watson AS, Mortensen M and Simon AK: Autophagy in the pathogenesis of myelodysplastic syndrome and acute myeloid leukemia. Cell Cycle 10: 1719-1725, 2011.

15. Mortensen M, Watson AS and Simon AK: Lack of autophagy in the hematopoietic system leads to loss of hematopoietic stem cell function and dysregulated myeloid proliferation. Autophagy 7: 1069-1070, 2011.

16. Ali AM and Raza A: Two different 'tales' of ATG7: Clinical relevance to myelodysplastic syndromes. Mol Cell Oncol 3: e1212686, 2016.

17. Jiang H, Yang L, Guo L, Cui N, Zhang G, Liu C, Xing L, Shao Z and Wang $\mathrm{H}$ : Impaired mitophagy of nucleated erythroid cells leads to anemia in patients with myelodysplastic syndromes. Oxid Med Cell Longev 2018: 6328051, 2018.

18. Jacquel A, Luciano F, Robert G and Auberger P: Implication and regulation of AMPK during physiological and pathological myeloid differentiation. Int J Mol Sci 19: E2991, 2018.

19. Zhao M and Klionsky DJ: AMPK-dependent phosphorylation of ULK1 induces autophagy. Cell Metab 13: 119-120, 2011.

20. Nieminen AI, Eskelinen VM, Haikala HM, Tervonen TA, Yan Y, Partanen JI and Klefstrom J: Myc-induced AMPK-phospho p53 pathway activates Bak to sensitize mitochondrial apoptosis. Proc Natl Acad Sci USA 110: E1839-E1848, 2013.

21. Livak KJ and Schmittgen TD: Analysis of relative gene expression data using real-time quantitative PCR and the 2(-Delta Delta C(T)) method. Methods 25: 402-408, 2001.

22. Klionsky DJ, Abdelmohsen K, Abe A, Abedin MJ, Abeliovich $\mathrm{H}$, Acevedo Arozena A, Adachi H, Adams CM, Adams PD, Adeli K, et al: Guidelines for the use and interpretation of assays for monitoring autophagy (3rd edition). Autophagy 12: 1-222, 2016.

23. Yim NH, Hwang $\mathrm{YH}$, Liang $\mathrm{C}$ and Ma JY: A platycoside-rich fraction from the root of Platycodon grandiflorum enhances cell death in A549 human lung carcinoma cells via mainly AMPK/mTOR/AKT signal-mediated autophagy induction. J Ethnopharmacol 194: 1060-1068, 2016.

24. Kim HI, Hong SH, Ku JM, Lim YS, Lee SJ, Song J, Kim TY, Cheon $\mathrm{C}$ and Ko SG: Scutellaria radix promotes apoptosis in non-small cell lung cancer cells via induction of AMPK-Dependent autophagy. Am J Chin Med 47: 691-705, 2019.

25. Wu Y, Wang X, Guo H, Zhang B, Zhang XB, Shi ZJ and Yu L: Synthesis and screening of 3-MA derivatives for autophagy inhibitors. Autophagy 9: 595-603, 2013.

26. Yin H, Yang X, Gu W, Liu Y, Li X, Huang X, Zhu X, Tao Y, Gou $X$ and He W: HMGB1-mediated autophagy attenuates gemcitabine-induced apoptosis in bladder cancer cells involving JNK and ERK activation. Oncotarget 8: 71642-71656, 2017.

27. Kim J, Kundu M, Viollet B and Guan KL: AMPK and mTOR regulate autophagy through direct phosphorylation of Ulk1. Nat Cell Biol 13: 132-141, 2011.

28. Xing JJ, Hou JG, Ma ZN, Wang Z, Ren S, Wang YP, Liu WC, Chen $\mathrm{C}$ and $\mathrm{Li}$ W: Ginsenoside $\mathrm{Rb} 3$ provides protective effects against cisplatin-induced nephrotoxicity via regulation of AMPK-/mTOR-mediated autophagy and inhibition of apoptosis in vitro and in vivo. Cell Prolif 52: e12627, 2019.

29. Sperling AS, Gibson CJ and Ebert BL: The genetics of myelodysplastic syndrome: From clonal haematopoiesis to secondary leukaemia. Nat Rev Cancer 17: 5-19, 2017. 
30. Schanz J, Tuchler H, Sole F, Mallo M, Luno E, Cervera J, Granada I, Hildebrandt B, Slovak ML, Ohyashiki K, et al: New comprehensive cytogenetic scoring system for primary myelodysplastic syndromes (MDS) and oligoblastic acute myeloid leukemia after MDS derived from an international database merge. J Clin Oncol 30: 820-829, 2012.

31. Li F, Zeng J, Gao Y, Guan Z, Ma Z, Shi Q, Du C, Jia J, Xu S, Wang X, et al: G9a inhibition induces autophagic cell death via AMPK/mTOR pathway in bladder transitional cell carcinoma. PLoS One 10: e0138390, 2015.

32. Mortensen M, Soilleux EJ, Djordjevic G, Tripp R, Lutteropp M, Sadighi-Akha E, Stranks AJ, Glanville J, Knight S, Jacobsen SE, et al: The autophagy protein Atg7 is essential for hematopoietic stem cell maintenance. J Exp Med 208: 455-467, 2011.

33. Hu X, Yan R, Cheng X, Song L, Zhang W, Li K and Zhao S: The function of sperm-associated antigen 6 in neuronal proliferation and differentiation. J Mol Histol 47: 531-540, 2016.

34. Li X, Xu L, Sun G, Wu X, Bai X, Li J, Strauss JF, Zhang Z and Wang H: Spag6 mutant mice have defects in development and function of spiral ganglion neurons, apoptosis, and higher sensitivity to paclitaxel. Sci Rep 7: 8638, 2017.

35. Maiuri MC, Criollo A and Kroemer G: Crosstalk between apoptosis and autophagy within the Beclin 1 interactome. EMBO J 29: 515-516, 2010.

36. Zhuang L, Ma Y, Wang Q, Zhang J, Zhu C, Zhang L and Xu X: Atg3 overexpression enhances bortezomib-induced cell death in SKM-1 cell. PLoS One 11: e0158761, 2016.

37. Romano A, Giallongo C, La Cava P, Parrinello NL, Chiechi A, Vetro C, Tibullo D, Di Raimondo F, Liotta LA, Espina V and Palumbo GA: Proteomic analysis reveals autophagy as pro-survival pathway elicited by long-term exposure with 5-azacitidine in high-risk myelodysplasia. Front Pharmacol 8: 204, 2017.

38. Gao L, Wang Z, Lu D, Huang J, Liu J and Hong L: Paeonol induces cytoprotective autophagy via blocking the Akt/mTOR pathway in ovarian cancer cells. Cell Death Dis 10: 609, 2019.
39. Maiuri MC, Zalckvar E, Kimchi A and Kroemer G: Self-eating and self-killing: Crosstalk between autophagy and apoptosis. Nat Rev Mol Cell Biol 8: 741-752, 2007.

40. Martelli AM, Evangelisti C, Chappell W, Abrams SL, Basecke J, Stivala F, Donia M, Fagone P, Nicoletti F, Libra M, et al: Targeting the translational apparatus to improve leukemia therapy: Roles of the PI3K/PTEN/Akt/mTOR pathway. Leukemia 25: 1064-1079, 2011.

41. Zeng J, Liu W, Fan YZ, He DL and Li L: PrLZ increases prostate cancer docetaxel resistance by inhibiting LKB1/AMPK-mediated autophagy. Theranostics 8: 109-123, 2018.

42. Li TT,Zhu D, Mou T, Guo Z, Pu JL, Chen QS, Wei XF and Wu ZJ: IL-37 induces autophagy in hepatocellular carcinoma cells by inhibiting the PI3K/AKT/mTOR pathway. Mol Immunol 87: 132-140, 2017.

43. Chan EY, Kir S and Tooze SA: siRNA screening of the kinome identifies ULK1 as a multidomain modulator of autophagy. J Biol Chem 282: 25464-25474, 2007.

44. Zheng Q, Zhao Y, Guo J, Zhao S, Fei C, Xiao C, Wu D, Wu L, $\mathrm{Li} X$ and Chang $\mathrm{C}$ : Iron overload promotes mitochondrial fragmentation in mesenchymal stromal cells from myelodysplastic syndrome patients through activation of the AMPK/MFF/Drp1 pathway. Cell Death Dis 9: 515, 2018.

45. Basiorka AA, McGraw KL, Eksioglu EA, Chen X, Johnson J, Zhang L, Zhang Q, Irvine BA, Cluzeau T, Sallman DA, et al: The NLRP3 inflammasome functions as a driver of the myelodysplastic syndrome phenotype. Blood 128: 2960-2975, 2016.

46. Bullón P, Alcocer-Gómez E, Carrión AM, Marín-Aguilar F, Garrido-Maraver J, Román-Malo L, Ruiz-Cabello J, Culic O, Ryffel B, Apetoh L, et al: AMPK phosphorylation modulates pain by activation of NLRP3 inflammasome. Antioxid Redox Signal 24: 157-170, 2016.

This work is licensed under a Creative Commons Attribution-NonCommercial-NoDerivatives 4.0 International (CC BY-NC-ND 4.0) License. 